INPLASY

PROTOCOL

To cite: Li et al. Helicobacter pylori reinfection and its risk factors after initial eradication:

A protocol for systematic review and meta-analysis. Inplasy protocol 202140121. doi:

10.37766/inplasy2021.4.0121

Received: 24 April 2021

Published: 24 April 2021

Corresponding author:

Renliang Li

1051545802@qq.com

Author Affiliation: Jiangxi University of Traditional Chinese Medicine

Support: Project for TCMST of Jiangxi.

Review Stage at time of this submission: The review has not yet started.

Conflicts of interest:

None declared.

\section{Helicobacter pylori reinfection and its risk factors after initial eradication: A protocol for systematic review and meta-analysis}

\author{
Li, RL1; Zhang, P2; Hu, ZY3; Yi, Y4; Chen, LS5; Zhang, HY6.
}

Review question / Objective: To confirm the global incidence of $\mathrm{H}$. pylori reinfection and systematically evaluate its risk factors.

Condition being studied: Helicobacter pylori (H. pylori) infection is a common health problem, which closely related to peptic ulcers, gastric cancer, and extragastric diseases. Drugs can successfully eradicate it. However, the recurrence of $\mathrm{H}$. pylori often occurs after initial eradication.

Information sources: We will search the following databases from their inception to November 2021: PubMed, Embase, Web of Science, the Cochrane Library, China National Knowledge Infrastructure, the Chongqing VIP Chinese Science and Technology Periodical Database, Wanfang Database, and China Biomedical Literature Database.

INPLASY registration number: This protocol was registered with the International Platform of Registered Systematic Review and Meta-Analysis Protocols (INPLASY) on 24 April 2021 and was last updated on 24 April 2021 (registration number INPLASY202140121).

\section{INTRODUCTION}

Review question / Objective: $\mathbf{X}$ To confirm the global incidence of $\mathrm{H}$. pylori reinfection and systematically evaluate its risk factors.
Condition being studied: Helicobacter pylori ( $\mathrm{H}$. pylori) infection is a common health problem, which closely related to peptic ulcers, gastric cancer, and extragastric diseases. Drugs can successfully eradicate it. However, the 
recurrence of $\mathbf{H}$. pylori often occurs after initial eradication.

\section{METHODS}

Participant or population: Patients with initial $H$. pylori infection were successfully eradicated.

Intervention: The change of $\mathbf{H}$. pylori from negative to positive at one year after initial eradication.

Comparator: H. pylori is still negative to positive at one year after initial eradication.

Study designs to be included: Cohort study or cross-sectional study.

Eligibility criteria: The study is considered qualified when the following criteria are met. (i) Cohort study or cross-sectional study; (ii) Patients with initial $H$. pylori infection were successfully eradicated; (iii) Age between 18 to 65 years-old.

Information sources: We will search the following databases from their inception to November 2021: PubMed, Embase, Web of Science, the Cochrane Library, China National Knowledge Infrastructure, the Chongqing VIP Chinese Science and Technology Periodical Database, Wanfang Database, and China Biomedical Literature Database.

Main outcome(s): The rate of $H$. pylori reinfection and its risk factors.

Quality assessment / Risk of bias analysis: Newcastle-Ottawa Scale (NOS) was applied to examine the methodological quality of the included studies. NOS had: 4 items for study subjects (4 points), 1 item for intergroup comparability ( 2 points), and 3 items for result measurement (3 points), with a total score of 9 (http://www.ohri.ca/ programs/clinical_epidemiology/oxford. asp).

Strategy of data synthesis: This metaanalysis will be conducted using STATA 14.0. A random-effects model will be used to estimate the pooled reinfection rate and its risk factors with $95 \% \mathrm{Cl}$. Heterogeneity will be assessed using a Chi-square test and 12 statistics ( $P$-value $<0.10$ or 12 over $50 \%$ were defined as substantial heterogeneity). Publication bias will be estimated by the Begg's test and Egger's test, with $\mathrm{P}<0.1$ indicating statistically significant.

Subgroup analysis: If the source of heterogeneity cannot be found after sensitivity analysis, we will do further subgroup analysis.

Sensitivity analysis: We will use the leaveone-out method for sensitivity analysis to judge the stability of outcome indicators.

Country(ies) involved: China.

Keywords: Helicobacter pylori, reinfection, protocol, systematic review, meta-analysis, risk factors.

Contributions of each author:

Author 1 - Renliang Li.

Author 2 - Ping Zhang.

Author 3 - Ziyi Hu.

Author 4 - Ying Yi - The author contributed to the development of the selection criteria, and the risk of bias assessment strategy.

Author 5 - Lisha Chen.

Author 6 - Hengyi Zhang. 